\section{EDUCATION}

Research, Inovotion and Solutions on-line
PSYCHOLOGY

I+D+i
Electronic Journal of Research

in Educational Psychology

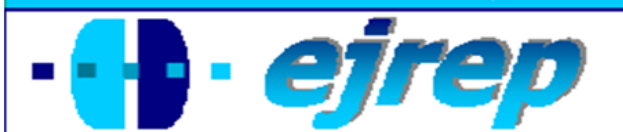

\title{
Comparación del desempeño social en niños con sordera profunda y audición normal, según distintos informantes
}

\section{María Julia Ipiña ${ }^{1}$, Leonardo Molina ${ }^{1}$, Rogelio Guzmán ${ }^{1}$, Cecilia Reyna ${ }^{2}$}

${ }^{1}$ Facultad de Psicología, Universidad Nacional de Córdoba, Córdoba

${ }^{2}$ Laboratorio de Psicología Cognitiva, Facultad de Psicología,

Universidad Nacional de Córdoba - CONICET, Córdoba

\section{Argentina}

Correspondencia: María Julia Ipiña. Bv. San Juan No 710 piso 12 depto. A, (5000) Córdoba, Argentina. E-mail: julipina83@hotmail.com

Este trabajo corresponde a parte de la tesis para optar por el grado de Licenciado en Psicología de María Julia Ipiña y Leonardo Molina, bajo la dirección del Lic. Rogelio Guzmán y Cecilia Reyna.

(C) Education \& Psychology I+D+i and Editorial EOS (Spain) 


\section{Resumen}

Introducción. A partir de considerar la relevancia de un óptimo desarrollo social en edades tempranas sumado a las particularidades que adquiere el mismo en los niños con sordera, este trabajo se propuso comparar la competencia social de niños con sordera profunda y audición normal en función del género y la edad, examinando también el rol del nivel socioeconómico.

Método. La muestra estuvo conformada por 32 niños con sordera profunda y 32 niños con audición normal, con edades comprendidas entre los 7 y 12 años que asistían a instituciones educativas y/o terapéuticas de la ciudad de Córdoba Capital (Argentina), también participaron los padres $(n=64)$ y docentes $(n=15)$. Los instrumentos utilizados fueron la escala MESSY en su versión para el docente y para el alumno y un cuestionario sociodemográfico aplicado a los tutores.

Resultados. Al considerar la valoración realizada por los niños se observaron diferencias significativas de grupo, presentando el grupo con sordera mayores niveles de inadecuación social. Mientras que según la valoración de los docentes, los niños con y sin sordera, presentan niveles semejantes de inadecuación social. Con respecto al nivel socioeconómico, no se observaron diferencias estadísticamente significativas.

Discusión. Se aprecian resultados diferenciales según el informante considerado. En términos generales, la sordera profunda no constituye un impedimento para el desarrollo de las habilidades sociales. Se discute la importancia de considerar otros factores contextuales y no sólo indicadores socioeconómicos.

Palabras Clave. Competencia social, sordera, escala MESSY, educación, desarrollo infantil. 


\title{
Comparison of social performance in children with profound deafness and children with normal hearing according to different informants
}

\begin{abstract}
Introduction. Considering the relevance of an optimal social development at early ages and its particularities in deaf children, this study aimed to compare the children's social competence with profound deafness and normal hearing depending on sex and age, examining also the role of socioeconomic status.

Method. Participants were 32 children with profound deafness and 32 children with normal hearing, from 7 to 12 years old, who were attending educational or therapeutic institutions of the city of Córdoba (Argentina), also participated parents $(n=64)$ and teachers $(n=15)$. The MESSY scale was used to assess social behavior in their versions for the teacher and for children, and also was applied a sociodemographic questionnaire to tutors.

Results. Considering the auto-evaluation were not significant differences of group, the group of children with deafness showed higher levels of social inadequacy. While according to the evaluation of teachers, children with and without deafness have similar levels of social inadequacy. With regard to socioeconomic status, were not observed statistically significant differences.
\end{abstract}

Discussion. The results were different according to the informant in question. In general, profound deafness is not an impediment to the development of social skills. We discuss the importance of considering other contextual factors, not just socioeconomic indicators.

Key words. Social competence, deafness, MESSY scale, education, child development.

Received: 09/13/10 Initial Acceptance: 09/21/10 Definitive Acceptance: 10/21/10 


\section{Introducción}

La pérdida de audición, ya sea total o parcial, resulta un tema de gran interés debido a la repercusión que puede llegar a tener en el desarrollo integral de la persona. Debido a ello, se requieren estudios que comprendan el período infantil, momento en el que se adquieren ciertas pautas de socialización, lo cual permitirá generar mejores prácticas de prevención e intervención.

En el presente artículo se hace hincapié en la sordera profunda, la cual implica la imposibilidad o dificultad de usar el sentido del oído debido a una pérdida de la capacidad auditiva parcial (hipoacusia) o total (cofosis) y unilateral o bilateral, pudiendo ser un rasgo hereditario o ser causada por una enfermedad, traumatismos, exposición a largo plazo al ruido o medicamentos agresivos para el nervio auditivo (ototóxicos). Específicamente, para que sea considerada sordera profunda debe existir una pérdida total o casi total de la capacidad auditiva, es decir, una pérdida en más de 85decibelios (dB) (Urbano y Estruch, 1996).

Durante las últimas décadas ha habido un gran avance en el estudio de los procesos comunicativos y en los contextos sociales en los cuales se desenvuelven los niños con sordera profunda. A partir de esto se ha generado un cambio de mirada en este tipo de problemáticas, pasando de una perspectiva clínico-terapéutica de la sordera, basada en el déficit, a una concepción basada en las capacidades. Al respecto, Skliar, Massone y Veinberg (1995) señalan dos modelos que buscan comprender esta cuestión según las implicancias educativas de la sordera, estos son:

- Modelo clínico-terapéutico: basado netamente en el déficit auditivo, el cual generaría todo tipo de problemáticas, ya sean comunicativas, sociales, cognitivas o lingüísticas; considera que se deben promover políticas educativas que estén exclusivamente ligadas a la enseñanza de la lengua oral.

- Modelo socio-antropológico: sostiene que el déficit auditivo de la persona sorda no afectará el posterior desarrollo, por lo que considera imprescindible la exposición temprana a la lengua de señas, promocionando a su vez políticas educativas que proporcionen información de manera bilingüe. Apunta a potenciar las posibilidades y no las deficiencias presuntamente impuestas por la sordera. 
En el marco del modelo socio-antropológico, este estudio pretende resaltar la importancia de potenciar las habilidades comunicativas propias de cada persona desde su más temprana infancia. Triado (1991) señala la relevancia de encontrar un intercambio gestual con el niño durante sus primeros meses de vida, debido a que en este período se adquiere el modo de comunicación con el mundo a través de un repertorio gestual que se acrecienta o encuentra su punto máximo con la adquisición del lenguaje; pero el principal recurso para adquirir la lengua oral es el sistema auditivo, el cual se encuentra disminuido o anulado por completo en los niños con sordera. La etapa de estimulación gestual pretende que el niño con sordera alcance una óptima retroalimentación con su entorno, considerando que la exposición temprana al lenguaje de señas facilitará el desarrollo social y cognitivo (Castro, 2003).

Los niños con sordera profunda constituyen un grupo extraordinariamente heterogéneo, por lo que resulta difícil realizar generalizaciones. A ello, se añade la influencia de múltiples variables que influyen en la evolución infantil, entre los que se encuentran el grado de pérdida auditiva, la etiología y la edad de comienzo de la sordera, como también los aspectos comunicativos y educativos. Dentro de éstos últimos se encuentran los factores que estimulan u obstaculizan el desarrollo, tanto por parte de los padres/tutores como de los docentes. Como factores estimuladores, se resalta en primera instancia la necesidad de la aceptación y preparación que posean tanto el propio niño como el entorno familiar para abordar la discapacidad, ya que como menciona Heiling (2001), los niños con sordera o con gran dificultad auditiva son competentes en tanto y en cuanto se les permita crecer en un ambiente comunicativo y lingüístico, en el cual sus capacidades sean respetadas y tenidas en cuenta. A partir de ello se desglosa la necesidad de insertar a los niños en modelos bilingües de comunicación, generando un espacio que permita al niño contactarse con los demás y emplear el lenguaje de signos junto a la lengua que utilizan las personas oyentes.

Al respecto, resulta de interés el estudio realizado por Carrada (2006) en el cual señala que la sordera no es la causa de la problemática, sino la falta de una correcta estimulación que favorezca el desarrollo cognitivo, social, afectivo y comunicativo. Por ello es de extrema importancia favorecer, estimular y ampliar el proceso de desarrollo de la madurez social para enriquecer el repertorio de comportamientos sociales válidos. Dichos comportamientos se enmarcan dentro del concepto de habilidades sociales, entendidas como repertorios de comportamientos que se presentan en la vida cotidiana y que contribuyen en forma decisiva para alcanzar buenos resultados en las relaciones interpersonales (Del Prette y Del Prette, 2002). 
Un concepto íntimamente relacionado a las habilidades sociales es el de competencia social, incluso numerosas veces son tratados como sinónimos. McFall (1982) señala que las habilidades sociales son comportamientos específicos que conducen a un desempeño social adaptativo, en tanto que la competencia social representa un término evaluativo, un juicio social realizado por padres, profesores o pares, valorándose si una persona ha realizado de forma adecuada determinadas tareas sociales. Por su parte, Fabes y Eisenberg (1999) definen a la competencia social como la habilidad para ser efectivo en lograr metas sociales constructivas (tener amigos, sostener interacciones, agradar, etc.), lo cual enfatiza, como señala Rendón (2007), los resultados sociales más que las conductas particulares. Cabe destacar que tanto las habilidades sociales como la competencia social se enmarcan dentro del comportamiento adaptativo de las personas.

Los comportamientos sociales adaptativos implican un proceso de aprendizaje que se desarrolla en la interacción con otras personas, y tal como señala Gresham (1981), es posibilitado por cuatro mecanismos: el aprendizaje por la experiencia, donde las habilidades sociales manifestadas son influenciadas por la maduración y las experiencias previas que modelan la conducta; el aprendizaje por modelado, que implica el aprendizaje por medio de la observación e imitación de otras personas; las instrucciones directas, mediante las cuales se aprenden determinadas habilidades o inhabilidades sociales; y por último, el feedback de otras personas, es decir la información que los demás nos dan con respecto a nuestra conducta.

En el desarrollo de las habilidades sociales, las relaciones interpersonales con los pares ocupan un lugar importante, tener amigos, pertenecer a un grupo, ser aceptado por los pares, entre otros, adquieren un carácter relevante en el período infantil y adolescente. El desenvolvimiento dentro de un grupo determina el tipo y la cantidad de relaciones sociales posibles de establecer, como lo demuestra un estudio realizado por Wauters y Knoors (2007), donde los niños populares mostraron muchos más comportamientos prosociales (ayudar, cooperar, ser considerado) y fueron más sociables (realizaban juegos asociativos, acercamientos amistosos y conversaciones sociales), evidenciando escasos comportamientos agresivos. A su vez, los niños que son rechazados, presentaron juegos agresivos y comportamientos antisociales (como intimidación o victimización) y eran considerados como arrogantes por sus pares. En cuanto a los niños con sordera profunda, estos autores señalan que cuentan con pocos amigos, menos interacciones con pares oyentes y son a menudo más relegados y descuidados por sus pares sin deficiencia auditiva, lo que posiblemente se deba a que una de las 
habilidades necesarias para interactuar con éxito con los compañeros oyentes se base en la lengua, área de un déficit inherente a la discapacidad de la sordera. Como indica Noll (2007), el lenguaje ocupa un rol central en el aprendizaje social y por lo tanto impacta en la capacidad del niño para poder aprender las habilidades necesarias para comunicarse con éxito, influyendo de este modo en sus comportamientos interpersonales.

Además de los factores familiares, el contexto más amplio en el que se desempeñan los niños también influye en su comportamiento social. En ese sentido, Murillo (2004) realiza un análisis de la influencia de las condiciones socioeconómicas sobre la educación y los procesos cognitivos. El autor rescata el planteamiento de Bruner (1976, citado en Murillo, 2004) respecto de las diferencias entre niños de clase media y de bajos recursos en cuanto a la forma, calidad e implementación del planteamiento de metas (motivación de logro). En relación al lenguaje, Bruner considera que tanto en los niveles socioeconómicos medios como bajos se logra manejar una amplitud idéntica de vocabulario y similares construcciones sintácticas. En las clases medias, el lenguaje se emplea como categorías formales que están reguladas por un conjunto de atribuciones criteriales, a partir de las cuales las cosas se clasifican sin ninguna referencia a su uso o reacción emocional producida. En cambio, en el lenguaje de los niños de clase socioeconómica baja, se utilizan categorías afectivas que están relacionadas con la reacción suscitada por los acontecimientos de su realidad. En tanto que, el desarrollo cognitivo estará relacionado con la calidad de la influencia de padres/tutores que son quienes establecen estilos de comunicación cognitivo racional con los niños. Esto es, la confianza transmitida, la señalización de los aciertos, el no castigo, los errores, el reforzamiento de conductas positivas y la búsqueda de soluciones (Murillo, 2004). A su vez, en las clases bajas existen elevados niveles de ansiedad ante el fracaso para solucionar problemas elementales de subsistencia, desconfianza y falta de estimulación para el logro de aspiraciones, lo cual se puede deber al nivel educativo y social.

En el ámbito nacional, se destaca el trabajo realizado por Carrada (2006), que se puede encuadrar en el modelo socio-antropológico, destacando, a su vez, la estimulación temprana para el logro de un intercambio comunicativo adecuado. La autora indagó acerca de la influencia de la lengua de señas en el desarrollo de las habilidades sociales en niños con sordera con edades comprendidas entre los 12 y 18 años de edad, encontrando que existen diferencias entre los niños oyentes y los que poseen deficiencia auditiva. Asimismo, estudios realizados en el ámbito internacional indican que los niños sordos tienden a inhibir y/o a limitar sus 
competencias lingüísticas y sociales cuando el contexto educativo no presenta las condiciones y recursos necesarios para abordar esta problemática (Antia y Kriemeyer, 2003).

Los estudios sobre la pérdida de audición, ya sea total o parcial, se han focalizado primordialmente en aspectos fisiológicos, siendo escasos los abordajes desde un plano psicológico. Un panorama semejante se observa en el plano nacional, en particular, en Córdoba (Argentina) son escasos los estudios que abordan esta temática. Debido a ello, y teniendo en cuenta la importancia del desarrollo social y el impacto que produce a corto y largo plazo, resulta de interés estudiar la competencia social en niños con sordera profunda. De esta manera, y a partir de la revisión antes expuesta, este trabajo se propuso comparar la competencia social de niños con sordera profunda y audición normal en función del género y la edad en el ámbito de la ciudad de Córdoba (Argentina). Además, considerar el rol del nivel socioeconómico en el desempeño social de los niños con sordera profunda.

La hipótesis central de este trabajo es que el desarrollo de la competencia social será dificultoso en los niños con sordera profunda debido a la demora o no adquisición de la lengua oral, por lo cual mostrarán un peor desempeño social en relación a niños que no tienen esta deficiencia. Además, teniendo en cuenta el rango etáreo comprendido, se espera que la edad no sea un factor influyente. Por el contrario, se hipotetiza que se observarán diferencias respecto del sexo, las mujeres mostrarán un mejor desempeño, diferencias de sexo que probablemente sean diferentes en el grupo de niños con sordera y en el grupo control. Por otra lado, se hipotetizan posibles diferencias en el comportamiento social de los niños con sordera a raíz del nivel socioeconómico. Finalmente, estas suposiciones estarán sujetas al informante (niño o docente), pudiendo no coincidir.

\section{Método}

\section{Participantes}

La muestra estuvo formada por 64 niños (71,9\% varones) con edades comprendidas entre los 7 y los 12 años, y también sus padres $(n=64)$ y docentes o profesionales $(n=15)$. Treinta y dos niños presentaban sordera profunda (grupo con sordera, GS) y asistían a tres instituciones especializadas de la ciudad de Córdoba (Argentina). Mientras que treinta y dos 
niños no tenían diagnóstico de sordera (grupo control, GC) y asistían a una escuela pública de la ciudad de Córdoba. Los niños de éste último grupo provenían de una muestra de 62 niños de dicha escuela, y fueron seleccionados siguiendo criterios de emparejamiento con el GS (sexo y edad), cuando resultaron más de dos participantes con características comunes a un participante del GS se procedió a seleccionar de manera aleatoria. No se observaron diferencias significativas entre los grupos con respecto a la edad $\left[t_{(62)}=1,196, p=.236\right]$. En la Tabla 1 se muestran las características demográficas (sexo, edad y nivel socioeconómico (NSE) ${ }^{1}$ ) de los niños de ambos grupos.

Con respecto a las características de los niños del GS, cabe señalar que el 78,1\% de los niños presentaba sordera desde el nacimiento, en tanto que el 21,9\% la adquirió entre los 2 y 36 meses de edad ( $M=13,43$ meses, $D T=13,66)$. En el 81,3\% de los casos eran los únicos que presentan esta disminución auditiva dentro de la familia nuclear. El 84,4\% de los niños sabía lengua de señas y el 75\% de las familias también. En las familias, el 71,9\% informó que utilizaban lengua de señas para comunicarse con el niño, mientras que el 53,1\% señaló emplear también otros medios de comunicación (modalidad oral, lenguaje de señas no convencional, lectura labial, escritura o por medio de indicaciones directas hacia objetos).

Tabla 1. Características demográficas de los niños

\begin{tabular}{|c|c|c|c|c|}
\hline & \multicolumn{2}{|c|}{$\begin{array}{c}\text { Grupo con Sordera } \\
\text { (GS) }\end{array}$} & \multicolumn{2}{|c|}{$\begin{array}{l}\text { Grupo Control } \\
\text { (GC) }\end{array}$} \\
\hline & $7-9$ años & $10-12$ años & $7-9$ años & $10-12$ años \\
\hline Varones & 8 & 15 & 8 & 15 \\
\hline Mujeres & 5 & 4 & 5 & 4 \\
\hline \multicolumn{5}{|l|}{$\mathrm{NSE}^{\mathrm{a}}$} \\
\hline $\begin{array}{c}\text { E a D2 - Marginal a bajo } \\
\text { inferior }\end{array}$ & \multicolumn{2}{|r|}{9} & \multicolumn{2}{|r|}{6} \\
\hline D1 - Bajo superior & \multicolumn{2}{|r|}{7} & \multicolumn{2}{|r|}{17} \\
\hline C3 - Medio bajo & \multicolumn{2}{|r|}{9} & \multicolumn{2}{|r|}{7} \\
\hline $\begin{array}{c}\mathrm{C} 2 \text { a C1- Medio medio a } \\
\text { medio alto }\end{array}$ & \multicolumn{2}{|r|}{6} & \multicolumn{2}{|r|}{2} \\
\hline
\end{tabular}

\footnotetext{
${ }^{1}$ EI NSE se determinó a partir de la relación de aportantes y miembros del hogar, nivel educativo, ocupación y cobertura de salud del principal sostén del hogar, e indicadores de indigencia (Comisión de Enlace Institucional, AAM-SAIMO-CEIM, 2006).
} 


\section{Instrumentos}

Cuestionario de datos sociodemográficos. Se recabó información sobre edad, sexo, NSE, nivel educativo de los padres, actividad del principal sostén del hogar, cantidad de habitantes por hogar, cobertura de salud y beneficio de planes sociales.

Cuestionario de información sobre el niño. Se indagó acerca de las características de la sordera del niño (tipo, modo de adquisición, uso de lengua de señas) y de las actividades recreativas realizadas (no se informan aquí).

Escala Matson para la Evaluación de Habilidades Sociales con Jóvenes (MESSY, Matson, Rotatori y Helsel, 1983). Se utilizaron las versiones de autoinforme (reducida) e informe del docente producto de estudios psicométricos previos realizados por Ipiña y Molina (2010) e Ipiña, Molina y Reyna (2010a) a partir de la versión en español de Trianes et al. (2002). La escala evalúa comportamientos sociales adaptativos y no adaptativos, considerando la relación del niño con sus pares y adultos a cargo; y en su versión original se puede aplicar a niños y jóvenes de 4 a 18 años. Cuenta con versiones para padres, docentes y alumnos, aunque en este estudio sólo se utilizaron las dos últimas. A continuación se describen las características de cada una de las escalas observadas en los estudios previos realizadas con muestras de niños sin alteraciones del desarrollo:

- MESSY-Autoinforme. Comprende un total de 62 ítems (alfa de Cronbach $(\alpha)=.81$ ) agrupados en 5 dimensiones: Agresividad/Conducta Antisocial (24 ítems, $\alpha=.85$ ), Habilidades Sociales Apropiadas (18 ítems, $\alpha=.79$ ), Amistad (9 ítems, $\alpha=.69$ ), Sobreconfianza/Celos/Soberbia (7 ítems, $\alpha=.65$ ), y Soledad/Ansiedad Social (4 ítems, $\alpha=.43$ ) (Ipiña, Molina \& Reyna, 2010b). Debido a la extensión de la escala, las autoridades escolares (principalmente de las instituciones donde asistían los niños con sordera) solicitaron que se aplique una versión reducida, la cual comprende 25 ítems y cuenta con adecuadas propiedades psicométricas, de manera semejante a la versión extensa (Ipiña \& Molina, 2010). La versión reducida se aplicó tanto a los niños del grupo con sordera (GS) como a los niños del grupo control (GC). En el presente estudio se examinó nuevamente la consistencia interna, obteniéndose los siguientes resultados: escala total $\alpha=.73$ (25 ítems), Agresividad/Conducta Antisocial $\alpha=.70$ (6 ítems), Habilidades Sociales Apropiadas $\alpha=.54$ (5 ítems), Amistad $\alpha=.70$ (5 ítems), Sobreconfianza/Celos/Soberbia $\alpha=.76$ (5 ítems), y Soledad/Ansiedad Social $\alpha=.68$ (4 ítems). 
- MESSY-Docente. Comprende un total de 64 ítems (alfa de Cronbach $(\alpha)=.95$ ) agrupados en 3 dimensiones: Agresividad/Conducta Antisocial (30 ítems, $\alpha=.97$ ), Habilidades Sociales Apropiadas (24 ítems, $\alpha=.92$ ), y Sobreconfianza/Celos/Soberbia (10 ítems, $\alpha=.95$ ) (Ipiña et al., 2010a). En el presente estudio se examinó nuevamente la consistencia interna, obteniéndose los siguientes resultados: escala total $\alpha=.93$, Agresividad/Conducta Antisocial $\alpha=.95$ (30 ítems); Habilidades Sociales Apropiadas $\alpha=.83$, y Sobreconfianza/Celos/Soberbia $\alpha=.90$.

En ambas versiones de la escala MESSY el comportamiento del niño se evalúa en una escala tipo Likert de 4 puntos $(1=$ Nunca, $2=$ A veces, $3=$ A menudo, $4=$ Siempre $)$. Mayor puntuación indica un nivel más alto de comportamientos inadecuados (dimensiones de comportamientos negativos) o adecuados socialmente (dimensiones de comportamientos positivos), en tanto que en la puntuación total refleja mayor inadecuación social. La puntuación de los ítems de cada dimensión positiva se deduce del máximo valor posible para esa dimensión y se suma a las dimensiones negativas. Por ejemplo, si una dimensión de comportamientos positivos comprende tres ítems, el máximo puntaje posible es 12 ; y suponiendo que un participante obtiene una puntuación en esa escala de 8 , el valor que se suma al resto de las dimensiones para obtener el índice de inadecuación social será igual a 4.

Si bien lo ideal sería valorar el comportamiento social de los niños del GS con instrumentos adaptados no sólo a la población local sino también a las particularidades de los niños con sordera, razones de orden práctico impidieron esa tarea en este trabajo. Sin embargo, cabe señalar que la versión original de la escala ha sido utilizada por Matson en varias oportunidades para estudiar diversas discapacidades y problemáticas, destacándose los estudios con jóvenes con sordera (Matson, Macklin y Helsel, 1985), jóvenes ciegos (Farkas, Sherick, Matson y Loebig, 1981) y con retraso mental (Matson, Carlisle y Bamburg, 1998).

\section{Procedimiento}

En primera instancia se contactó al personal directivo con el fin de solicitar la autorización de ingreso a los establecimientos, explicando fines y modos de realización de la actividad, y se contactó a los docentes y/o profesionales participantes. Luego, se solicitó el consentimiento informado a padres y/o tutores, junto con el cuestionario sociodemográfico y el cuestionario de información del niño. La aplicación de la escala MESSY-Autoinforme a los niños del GS se realizó en grupos reducidos $(n=4)$ con la presencia de un intérprete, lo cual fue un 
requisito de las instituciones para poder llevar a cabo la toma de los cuestionarios, señalando que la comunicación se vería imposibilitada si no se contase con un experto en lengua de señas. Asimismo, los profesionales a cargo de los niños colaboraron con la tarea en los casos que fue necesario, pero siempre ante la presencia y control general de los investigadores. Por otra parte, la aplicación a los niños del GC se realizó en un aula escolar en grupos pequeños $(n=4)$. Finalmente, los docentes y profesionales informaron acerca del comportamiento social de los niños participantes a través de la escala MESSY-Docente, tarea que cumplimentaron de forma paralela al momento en que los investigadores aplicaban los cuestionarios a los niños.

\section{Diseño y análisis estadístico}

Se realizó un estudio ex post facto prospectivo factorial, las variables no fueron manipuladas sino que las variaciones se daban de manera natural (Montero y León, 2007). Los datos fueron analizados con el paquete estadístico SPSS 17. Primero se examinaron los datos de forma exploratoria con el fin de identificar el comportamiento de casos y variables. Luego, se comparó el comportamiento social de los niños mediante análisis de la varianza (ANOVA) a tres vías de clasificación: grupo (con sordera - control), sexo (masculino - femenino) y grupo etáreo ( 7 a 9 años - 10 a 12 años). También se emplearon pruebas $t$ para muestras independientes para comparar el desempeño social en función del grupo (con sordera - control). Finalmente, se estudió el comportamiento social de los niños con sordera según el NSE a través de ANOVAs.

\section{Resultados}

Previo al examen de diferencias de medias del comportamiento social de los niños del GS y GC, se analizaron de manera exploratoria los datos. No se identificaron casos atípicos univariados ni multivariados (Tabachnick y Fidell, 2001) en las variables referidas a comportamiento social. El análisis descriptivo mostró que todas las variables comportamentales presentaban valores de asimetría y curtosis dentro del rango \pm 1.5 , lo cual indica distribución normal. 
Para comparar el comportamiento social de los niños se llevaron a cabo análisis de la varianza (ANOVA) a tres vías: grupo (con sordera - control), sexo (masculino - femenino) y grupo etáreo (7 a 9 años - 10 a 12 años). Las variables dependientes fueron las puntuaciones totales y de cada una de las dimensiones de las versiones para el docente y de autoinforme del MESSY. En las Tablas 2 y 3 se muestran los estadísticos descriptivos. Debido a que se realizaron ANOVAs separados sobre cada variable dependiente, para controlar el error Tipo I se aplicó corrección de Bonferroni, la cual implica ajustar el nivel alfa según el número de contrastes (Tabachnick y Fidell, 2001). El nivel de significación ajustado fue de .005 (alfa total / número de contrastes, .05 / 10). Con respecto a la versión del MESSY-Autoinforme (Tabla 4) no se observaron interacciones significativas, pero sí efectos significativos de grupo en la puntuación total $\left[F_{(1,56)}=25,28, p=.000, \eta^{2}=.31\right]$ y en las siguientes dimensiones: Sobreconfianza/Celos/Soberbia $\left[F_{(1,56)}=12,60, p=.000, \eta^{2}=.18\right]$ y Soledad/Ansiedad Social $\left[F_{(1,56)}=\right.$ $\left.18,30, p=.000, \eta^{2}=.25\right]$. El GS presentó las mayores puntuaciones, lo cual indica que los niños con sordera presentan mayores niveles de inadecuación social. El porcentaje de varianza de las variables dependientes atribuible a la variable grupo fue en estos casos del $18 \%$ al $31 \%$. En las dimensiones positivas, Habilidades Sociales Apropiadas y Amistad, las puntuaciones medias del GS fueron sutilmente menores al GC, sin alcanzar significación estadística.

Al considerar la versión MESSY-Docente (Tabla 5) no se observaron interacciones significativas y tampoco efecto de grupo, es decir que a partir de la valoración de los docentes, los niños con y sin sordera presentan niveles semejantes de inadecuación social. En cambio, sí se observó un efecto principal significativo de sexo en dos dimensiones: Agresividad/Conducta Antisocial $\left[F_{(1,56)}=8,81, p=.004, \eta^{2}=.14\right]$ y Sobreconfianza/Celos/Soberbia $\left[F_{(1,56)}=9,34, p=.003, \eta^{2}=.14\right]$. Los varones mostraron las puntuaciones más altas. En este caso, el porcentaje de varianza de las variables dependientes atribuible a la variable sexo fue del 14\%. Sin embargo, tanto según el autoinforme como el informe del docente, no resultaron significativas las interacciones entre sexo y grupo, y tampoco las interacciones y efectos principales que implicaban la edad (al nivel de significación establecido en .005).

Los supuestos de normalidad y homogeneidad de varianzas se verificaron a través de gráficos ( $q-q$ plot, gráfico de residuos versus predichos) y pruebas estadísticas (prueba de Shapiro-Wilks y prueba de Levene). En todos los casos se cumplió el supuesto de normalidad. En tanto que el supuesto de varianzas homogéneas sólo se incumplió para la dimensión Sole- 
dad/Ansiedad Social de la versión del alumno, en ese caso se aplicó una transformación inversa, siguiendo las recomendaciones de Hair, Anderson, Tatham y Black (1999). El reducido número de casos y su consecuente efecto sobre la potencia, llevan a considerar no concluyentes los resultados no significativos (Aron y Aron, 2001).

Como una forma de esclarecer el efecto del tamaño de las muestras sobre los resultados y considerando los resultados de los ANOVAs a tres vías de clasificación, se llevaron a cabo nuevos análisis a los fines de valorar las diferencias entre el GS y el GC colapsando los factores sexo y edad cuando éstos no fueron significativos, por lo cual se utilizaron pruebas $t$ para muestras independientes y ANOVAs a dos vías de clasificación. Los resultados obtenidos fueron semejantes a los antes reportados, sin nuevos resultados significativos a nivel estadístico.

Tabla 2. Media y desviación típica del MESSY-Autoinforme según grupo, sexo y edad

\begin{tabular}{|c|c|c|c|c|c|c|c|c|}
\hline & & & Total & F1 & F2 & F3 & F4 & F5 \\
\hline \multirow{4}{*}{$\begin{array}{l}\text { Grupo } \\
\text { con } \\
\text { Sordera }\end{array}$} & \multirow[t]{2}{*}{ Varones } & $\begin{array}{c}7-9 \text { años } \\
(n=8)\end{array}$ & $\begin{array}{l}40,63 \\
(3,58)\end{array}$ & $\begin{array}{c}9,63 \\
(3,42)\end{array}$ & $\begin{array}{l}13,63 \\
(4,37)\end{array}$ & $\begin{array}{l}13,63 \\
(3,74)\end{array}$ & $\begin{array}{l}16,50 \\
(2,88)\end{array}$ & $\begin{array}{c}7,50 \\
(3,12)\end{array}$ \\
\hline & & $\begin{array}{c}10-12 \text { años } \\
(n=15)\end{array}$ & $\begin{array}{l}39,67 \\
(9,12)\end{array}$ & $\begin{array}{l}11,93 \\
(4,23)\end{array}$ & $\begin{array}{l}12,73 \\
(3,92)\end{array}$ & $\begin{array}{l}16,47 \\
(1,92)\end{array}$ & $\begin{array}{l}17,00 \\
(2,20)\end{array}$ & $\begin{array}{c}8,47 \\
(3,04)\end{array}$ \\
\hline & \multirow[t]{2}{*}{ Mujeres } & $\begin{array}{c}7-9 \text { años } \\
(n=5)\end{array}$ & $\begin{array}{l}41,80 \\
(6,72)\end{array}$ & $\begin{array}{c}9,80 \\
(2,49)\end{array}$ & $\begin{array}{l}10,60 \\
(3,36)\end{array}$ & $\begin{array}{l}13,60 \\
(5,37)\end{array}$ & $\begin{array}{l}14,60 \\
(4,51)\end{array}$ & $\begin{array}{c}9,60 \\
(1,95)\end{array}$ \\
\hline & & $\begin{array}{c}10-12 \text { años } \\
(n=4)\end{array}$ & $\begin{array}{l}49,25 \\
(6,70)\end{array}$ & $\begin{array}{l}15,00 \\
(2,94)\end{array}$ & $\begin{array}{l}14,50 \\
(2,52)\end{array}$ & $\begin{array}{l}16,00 \\
(3,37)\end{array}$ & $\begin{array}{l}16,25 \\
(4,11)\end{array}$ & $\begin{array}{l}12,00 \\
(3,16)\end{array}$ \\
\hline \multirow[t]{4}{*}{$\begin{array}{l}\text { Grupo } \\
\text { Control }\end{array}$} & \multirow[t]{2}{*}{ Varones } & $\begin{array}{c}7-9 \text { años } \\
(n=8)\end{array}$ & $\begin{array}{l}26,38 \\
(3,74)\end{array}$ & $\begin{array}{c}6,75 \\
(1,28)\end{array}$ & $\begin{array}{c}9,12 \\
(3,18)\end{array}$ & $\begin{array}{l}15,87 \\
(3,00)\end{array}$ & $\begin{array}{l}19,13 \\
(1,46)\end{array}$ & $\begin{array}{c}5,50 \\
(1,07)\end{array}$ \\
\hline & & $\begin{array}{c}10-12 \text { años } \\
(n=15)\end{array}$ & $\begin{array}{l}35,13 \\
(8,58)\end{array}$ & $\begin{array}{c}9,00 \\
(3,23)\end{array}$ & $\begin{array}{l}11,93 \\
(4,61)\end{array}$ & $\begin{array}{l}15,13 \\
(3,76)\end{array}$ & $\begin{array}{l}17,00 \\
(3,44)\end{array}$ & $\begin{array}{c}6,33 \\
(2,09)\end{array}$ \\
\hline & \multirow[t]{2}{*}{ Mujeres } & $\begin{array}{c}7-9 \text { años } \\
(n=5)\end{array}$ & $\begin{array}{l}36,20 \\
(8,17)\end{array}$ & $\begin{array}{c}9,40 \\
(4,39)\end{array}$ & $\begin{array}{l}12,20 \\
(3,96)\end{array}$ & $\begin{array}{l}14,60 \\
(3,13)\end{array}$ & $\begin{array}{l}17,80 \\
(1,48)\end{array}$ & $\begin{array}{c}7,00 \\
(2,45)\end{array}$ \\
\hline & & $\begin{array}{c}10-12 \text { años } \\
(n=4)\end{array}$ & $\begin{array}{l}31,50 \\
(5,74)\end{array}$ & $\begin{array}{c}7,75 \\
(1,71)\end{array}$ & $\begin{array}{c}8,75 \\
(0,96)\end{array}$ & $\begin{array}{l}16,25 \\
(3,50)\end{array}$ & $\begin{array}{r}13,50 \\
(3,70)\end{array}$ & $\begin{array}{c}4,75 \\
(0,96)\end{array}$ \\
\hline
\end{tabular}

Nota. Total $=$ puntuación total MESSY-Autoinforme; F1 = factor 1, Sobreconfianza/Celos/ Soberbia; F2 = factor 2, Agresividad/Conducta Antisocial; F3 = factor 3, Habilidades Sociales Apropiadas; F4 = factor 4, Amistad; F5 = factor 5, Soledad/Ansiedad Social. Entre paréntesis se muestra la desviación típica. 
Tabla 3. Media y desviación típica del MESSY-Docente según grupo, sexo y edad

\begin{tabular}{|c|c|c|c|c|c|c|}
\hline & & & Total & F1 & F2 & F3 \\
\hline \multirow{4}{*}{$\begin{array}{l}\text { Grupo } \\
\text { con } \\
\text { Sordera }\end{array}$} & \multirow[t]{2}{*}{ Varones } & $\begin{array}{c}7 \text { - } 9 \text { años } \\
(n=8)\end{array}$ & $\begin{array}{l}130,88 \\
(22,11)\end{array}$ & $\begin{array}{c}71,38 \\
(13,84)\end{array}$ & $\begin{array}{c}60,12 \\
(11,38)\end{array}$ & $\begin{array}{l}23,63 \\
(6,41)\end{array}$ \\
\hline & & $\begin{array}{c}10-12 \text { años } \\
(n=15)\end{array}$ & $\begin{array}{l}114,47 \\
(27,52)\end{array}$ & $\begin{array}{c}63,13 \\
(14,18)\end{array}$ & $\begin{array}{c}67,20 \\
(11,48)\end{array}$ & $\begin{array}{l}22,53 \\
(5,89)\end{array}$ \\
\hline & \multirow[t]{2}{*}{ Mujeres } & $\begin{array}{c}7 \text { - } 9 \text { años } \\
(n=5)\end{array}$ & $\begin{array}{c}93,20 \\
(19,54)\end{array}$ & $\begin{array}{c}46,80 \\
(10,26)\end{array}$ & $\begin{array}{c}66,80 \\
(12,01)\end{array}$ & $\begin{array}{l}17,20 \\
(4,38)\end{array}$ \\
\hline & & $\begin{array}{c}10-12 \text { años } \\
(n=4)\end{array}$ & $\begin{array}{l}115,50 \\
(20,86)\end{array}$ & $\begin{array}{c}63,00 \\
(23,34)\end{array}$ & $\begin{array}{c}67,00 \\
(11,86)\end{array}$ & $\begin{array}{l}23,50 \\
(9,75)\end{array}$ \\
\hline \multirow[t]{4}{*}{$\begin{array}{l}\text { Grupo } \\
\text { Control }\end{array}$} & \multirow[t]{2}{*}{ Varones } & $\begin{array}{c}7 \text { - } 9 \text { años } \\
(n=8)\end{array}$ & $\begin{array}{l}134,75 \\
(22,31)\end{array}$ & $\begin{array}{c}70,25 \\
(14,64)\end{array}$ & $\begin{array}{l}61,25 \\
(5,31)\end{array}$ & $\begin{array}{l}29,75 \\
(6,30)\end{array}$ \\
\hline & & $\begin{array}{c}10-12 \text { años } \\
(n=15)\end{array}$ & $\begin{array}{l}122,60 \\
(28,85)\end{array}$ & $\begin{array}{c}65,47 \\
(16,37)\end{array}$ & $\begin{array}{l}64,07 \\
(8,98)\end{array}$ & $\begin{array}{l}25,20 \\
(6,13)\end{array}$ \\
\hline & \multirow[t]{2}{*}{ Mujeres } & $\begin{array}{c}7-9 \text { años } \\
(n=5)\end{array}$ & $\begin{array}{l}120,20 \\
(26,81)\end{array}$ & $\begin{array}{c}62,20 \\
(17,12)\end{array}$ & $\begin{array}{c}61,00 \\
(10,07)\end{array}$ & $\begin{array}{l}23,00 \\
(7,25)\end{array}$ \\
\hline & & $\begin{array}{c}10-12 \text { años } \\
(n=4)\end{array}$ & $\begin{array}{c}94,25 \\
(24,68)\end{array}$ & $\begin{array}{c}45,75 \\
(18,34)\end{array}$ & $\begin{array}{l}63,00 \\
(9,63)\end{array}$ & $\begin{array}{l}15,50 \\
(5,57)\end{array}$ \\
\hline
\end{tabular}

Nota . Total $=$ puntuación total MESSY-Docente; F1 = factor 1, Agresividad/Conducta Antisocial; F2 = factor 2, Habilidades Sociales Apropiadas; F3 = factor 3, Sobreconfianza/Celos/Soberbia. Entre paréntesis se muestra la desviación típica.

Tabla 4. Resultados de los ANOVAs para el MESSY-Autoinforme según grupo, sexo y edad

\begin{tabular}{lcccccc}
\hline & Total & F1 & F2 & F3 & F4 & F5 \\
\hline Grupo $(1,56)$ & $25,28 * * *$ & $12,60 * * *$ & $4,60 *$ & 0,32 & 0,84 & $18,30 * * *$ \\
Sexo $(1,56)$ & $4,09 *$ & 1,50 & 0,10 & 0,03 & $4,99 *$ & 1,88 \\
Edad $(1,56)$ & 1,59 & 4,58 & 0,29 & 2,60 & 1,63 & 0,00 \\
Grupo X Sexo $(1,56)$ & 0,30 & 0,24 & 0,07 & 0,01 & 0,42 & 3,58 \\
Grupo X Edad $(1,56)$ & 0,08 & 3,32 & 0,69 & 1,29 & $6,57 *$ & 1,96 \\
Sexo X Edad (1,56) & 0,36 & 0,07 & 0,11 & 2,61 & 0,09 & 1,49 \\
Grupo X Sexo X Edad $(1,56)$ & $6,81 *$ & 3,21 & $6,30 *$ & 0,55 & 0,99 & 1,45 \\
\hline
\end{tabular}

Nota. Total $=$ puntuación total MESSY-Autoinforme; F1 $=$ factor 1 , Sobreconfianza/Celos/ Soberbia; F2 = factor 2, Agresividad/Conducta Antisocial; F3 = factor 3, Habilidades Sociales Apropiadas; F4 = factor 4, Amistad; F5 = factor 5, Soledad/Ansiedad Social. Entre paréntesis se muestran los grados de libertad.

${ }^{*} p<.05 . * * p<.01 . * * * p<.005$.

Finalmente, con el fin de examinar si había diferencias en el comportamiento social de los niños con sordera en función del NSE se llevaron a cabo ANOVAs para cada una de las variables de comportamiento social (docente y alumno). Los niveles del NSE fueron cuatro: marginal a bajo inferior (E a D2), bajo superior (D1), medio bajo (C3) y medio a medio alto 
(C2 a C1). En ningún caso se observaron resultados estadísticamente significativos al nivel de significación ajustado (.005) y tampoco a un nivel de significación menor (.05). Cabe señalar que en la muestra de niños con sordera no estuvo representado el NSE más alto (AB), por lo que resta considerar ese nivel.

Tabla 5. Resultados de los ANOVAs para el MESSY-Docente según grupo, sexo y edad

\begin{tabular}{lcccc}
\hline & Total & F1 & F2 & F3 \\
\hline Grupo $(1,56)$ & 0.00 & 1.06 & 0.85 & 0.00 \\
Sexo $(1,56)$ & $8.81^{* * *}$ & 0.20 & $9.34^{* * * *}$ & $8.81^{* * *}$ \\
Edad $(1,56)$ & 0.56 & 1.11 & 0.91 & 0.56 \\
Grupo X Sexo $(1,56)$ & 0.03 & 0.46 & 2.35 & 0.03 \\
Grupo X Edad $(1,56)$ & 2.43 & 0.05 & $5.80^{*}$ & 2.43 \\
Sexo X Edad $(1,56)$ & 0.52 & 0.45 & 0.38 & 0.52 \\
Grupo X Sexo X Edad $(1,56)$ & 4.17 & 0.28 & 2.08 & 4.17 \\
\hline
\end{tabular}

Nota. Total $=$ puntuación total MESSY-Docente; F1 = factor 1, Agresividad/Conducta Antisocial; F2 = factor 2, Habilidades Sociales Apropiadas; F3 = factor 3, Sobreconfianza/Celos/ Soberbia. Entre paréntesis se muestran los grados de libertad.

${ }^{*} p<.05 .{ }^{* *} p<.01 .{ }^{* * *} p<.005$.

\section{Discusión y conclusiones}

Este trabajo se propuso comparar el desempeño social de niños con sordera profunda y audición normal, considerando también los factores sexo y edad. Al considerar el autoinforme, se observaron diferencias en algunas dimensiones del comportamiento social, los niños con sordera mostraron mayores niveles de inadecuación social en Sobreconfianza/Celos/Soberbia y Soledad/Ansiedad Social, y también al considerar la puntuación total.

Sin embargo, no se apreciaron diferencias respecto del grupo según el informe de los docentes, tanto los niños con sordera como el grupo control parecen contar con niveles semejantes de inadecuación social. Aunque sí existieron diferencias en el registro realizado por los docentes con respecto al sexo en las dimensiones de Agresividad/Conducta Antisocial y Sobreconfianza/Celos/Soberbia, mostrando los varones mayores índices de inadecuación social.

Numerosos estudios apoyan las diferencias de sexo en el comportamiento social (Crick y Grotpeter, 1995; Ison, 2001, entre otros), lo cual también se evidenció en este traba- 
jo, sin embargo, no se encontraron efectos de interacción entre el grupo y el sexo. Esto está en línea con lo observado por Carrada (2006), quién reportó que alumnas sordas resultaron ser más asertivas y alumnos sordos más agresivos al interactuar con pares. Según la autora, el género en sí es un factor diferencial, independientemente de la variable sordera, ya que socialmente los niños presentan conductas agresivas e impulsivas en mayor proporción que las niñas, por lo que considera que la sordera no constituye un impedimento para el desarrollo de las habilidades sociales sino que están determinadas por el género de la persona.

$\mathrm{Al}$ considerar los resultados referidos al NSE resulta llamativa la ausencia de diferencias significativas en el desempeño social de los niños con sordera en función del mismo. En diversos estudios el factor económico ha mostrado una importante incidencia. Tal es el caso del trabajo desarrollado por Trianes, Cardelle-Elawar, Blanca y Muñoz (2003), donde las diferencias en el comportamiento social fueron más notorias al considerar el contexto socioeconómico que el género, manifestando los niños de contextos más desfavorecidos un mejor desempeño social según su autovaloración; ello condujo a los autores a interpretar esos resultados como función de diferentes criterios culturales que se emplean para juzgar un comportamiento socialmente habilidoso. La revisión de Chen y French (2008) remarca la relevancia de una perspectiva contextual del desarrollo, en la cual los procesos de interacción social sirven para mediar la influencia en el desarrollo humano. Si bien en nuestro trabajo los resultados no fueron significativos en relación al NSE, es fundamental tener en cuenta la importancia del contexto inmediato del niño, el cual se espera que favorezca, estimule y amplíe el proceso de desarrollo de la madurez social para preparar a los niños sordos en las complejas interacciones humanas (Carrada 2006).

En síntesis, sólo considerando el informe de los niños fue posible identificar diferencias entre los niños del grupo con sordera y el grupo control, en detrimento de los niños con sordera en algunas dimensiones y en la puntuación total de comportamiento social. Sin embargo, no se apreciaron interacciones significativas entre grupo, sexo y edad. Por otra parte, con respecto a los docentes/profesionales que valoraron el comportamiento de los niños cabe destacar que pertenecían a instituciones distintas, y cada uno valoró a los niños de su institución.

A partir de estos resultados se hipotetizan posibles influencias del contexto de evaluación en los resultados obtenidos a partir del informe de los docentes/profesionales, convier- 
tiéndose aquí en desventaja el criterio comparativo que caracteriza a estos informantes (Canivez y Rains, 2002). Es probable que cada uno de ellos responda basándose en las distintas disciplinas que ejercen, es decir, desde la docencia, fonoaudiología y psicología, lo cual determinaría la subjetividad con la cual se evalúa cada comportamiento, ya que cada uno se posicionaría desde distintos ángulos en el momento de considerar una conducta como competente socialmente. Estos resultados invitan a emplear nuevas estrategias analíticas que comprendan los aspectos mencionados. Una vía posible es emplear abordajes interdisciplinarios que permitan lograr una unificación de conceptos y en función de ello alcanzar intervenciones más integrales y complejas. Asimismo, es recomendable incorporar información respecto de la experiencia profesional de los participantes, factor que no se tuvo en cuenta en este trabajo y que según indican algunos estudios parece ejercer un efecto moderador en la valoración del comportamiento social (Gotzens, Badia, Genovard y Dezcallar, 2010). Por otra parte, estudios futuros podrían incluir el informe de padres o tutores, teniendo en cuenta que son los principales encargados de la socialización primaria y podrían brindar mayor información del comportamiento en otros contextos, logrando de este modo un estudio más completo sobre el desarrollo de la competencia social en los niños.

Como describe Heiling (2001), los niños sordos son competentes en tanto y en cuanto se les permita crecer en un ambiente propicio para el desarrollo de la comunicación, respetando sus capacidades particulares. Estos aportes describen principalmente la necesidad de un ambiente contenedor, generador de oportunidades y no de limitaciones u obstáculos. Por lo cual se estima que además de las condiciones socioeconómicas, es de importancia considerar la atención y participación de la familia en esta problemática, favoreciendo y permitiendo que el niño se desarrolle en un ambiente óptimo.

A partir de este estudio, una de las cuestiones fundamentales a tener en cuenta es el respeto y la consideración del lenguaje de señas como la lengua fundamental de las personas con deficiencias auditivas, ya que de ese modo se simplifica el intercambio comunicativo con el resto de la población, facilitando el desarrollo de la competencia social. Por otro lado, surge el interrogante de si estamos preparados como profesionales y ciudadanos para afrontar este y otros tipos de discapacidades. Como fue expuesto a lo largo de este trabajo, desde hace décadas las investigaciones se centran exclusivamente en las incapacidades sin tener en cuenta cuáles son las capacidades que poseen las personas. Conocer y comprender las capacidades es 
un paso fundamental para lograr una adecuada estimulación de funciones compensatorias, y en términos generales, para el óptimo desarrollo de cada individuo.

\section{Referencias}

Antia, S. D. y Kreimeyer, K. (2003). Peer interactions of deaf and hard of hearing children. In M. Marschark \& P. E. Spencer (Eds.), Oxford Handbook of deaf studies, language, and education (pp. 164 -176). New York: Oxford University Press.

Aron, A. y Aron, E. N. (2001). Estadística para psicología (2da ed.). Buenos Aires: Pearson Education.

Canivez, G. L. y Rains, J. D. (2002). Construct validity of the adjustment scales for children and adolescent and the preschool and kindergarten behavior scales: convergent and divergent evidence. Psychology in the Schools, 39(6), 621-633.

Carrada, M. (2006). La influencia de la lengua de señas en el adecuado desarrollo de las habilidades sociales. Revista Irice, 19, 119-125.

Castro, P. (2003) Aprendizaje del lenguaje en niños sordos: fundamentos para la adquisición temprana de lenguaje de señas. Journal of Deaf Studies and Deaf Education, 13(1), 21-36.

Comisión de Enlace Institucional, AAM-SAIMO-CEIM (2006). Nivel Socio Económico 2006. Recuperado en diciembre de 2008, de http:/www.saimo.org.ar/socios/Socios/NSE 2006-23nov2006-Informe_final.pdf.

Crick, N. R. y Grotpeter, J. K. (1995). Relational aggression, gender, and social-psychological adjustment. Child Development, 66(3), 710-722.

Chen, X. y French, D. (2008) Children's social competence in cultural context. Journal the Annual Review Psychology, 59, 591-616.

Del Prette, Z. A. y Del Prette, A. (2002). Psicología de las Habilidades Sociales: Terapia y Educación. México: Manual Moderno.

Fabes, R., y Eisenberg, N. (1999). Regulation, emotionality, and preschoolers' socially competent peer interactions. Child Development, 70(2), 432. 
Farkas, G. M., Sherick, R., Matson, J. L. y Loebig, M. (1981). Skills training of a blind child via differential reinforcement. Behavior Therapist, 4, 24-26.

Gotzens, C., Badia, M., Genovard, C. y Dezcallar, T. (2010). Estudio comparativo de la gravedad atribuida a las conductas disruptivas en el aula. Electronic Journal of Research in Educational Psychology, 8(1), 33-58.

Gresham, F. (1981). Validity of social skills measures for assessing the social competence in low status children: a multivariate investigation. Developmental Psychology, 17, 390398.

Hair, J. F., Anderson, R. E., Tatham, R. L. y Black, W. C. (1999). Análisis multivariante (5ta ed.). Madrid: Prentice Hall.

Heiling, K. (2001). Review of papers presented at the round Table session on social and emotional development in deaf children: services in four countries. Scandinavian Audiology, 30(2), 101-109.

Ipiña, M. J. y Molina, L. (2010). Competencia social en niños con sordera profunda (Tesis de grado no publicada). Facultad de Psicología, Universidad Nacional de Córdoba, Argentina.

Ipiña, M. J., Molina, L. y Reyna, C. (2010a). Propiedades psicométricas de la Escala MESSY (versión docente) en una muestra de niños argentinos. Artículo enviado para su publicación.

Ipiña, M. J., Molina, L. y Reyna, C. (2010b). Características psicométricas de la Escala MESSY (version auto-informe) en una muestra de niños cordobeses. Clínica e Investigación. Contribuciones a las Problemáticas Sociales, 461-464.

Ison, M. S. (2001). Training in social skills: An alternative technique for handling disruptive child behavior. Perceptual and Motor Skills, 88, 903-911.

Matson, J. L., Carlisle, C. y Bamburg, L. (1998). The convergent validity of the Matson Evaluation of Social Skills for Individuals with Severe Retardation (MESSIER). Research in Developmental Disabilities, 19(6), 493-500.

Matson, J. L., Macklin, G. F. y Helsel, W. J. (1985). Psychometric properties of The Matson Evaluation of Social Skills with Youngsters (MESSY) with emotional problems and self concept in deaf children. Journal of Behavior Therapy \& Experimental Psychiatry, 16(2), 117-124. 
Matson, J. L., Rotatori, A. F., y Helsel, W. J. (1983). Development of a rating scale of to measure social skills in children: The Matson Evaluation of Social Skills with Youngsters (MESSY). Behaviour Research and therapy, 21, 335-340.

Montero, I. y León, O. G. (2007). Guía para nombrar los estudios de investigación en Psicología. International Journal of Clinical and Health Psycholog, 7(3), 847-862.

Murillo, C. (2004). Infancia, clase social y estilos cognitivos: Una discusión desde la psicología. Perspectivas en Psicologia, 6, 51-58.

McFall, R. (1982). A review and reformulation of the concept of social skills. Behavioral Assessment, 4(1), 1-33.

Noll, D. L. (2007). Activities for social skills development in deaf children preparing to enter the mainstream. Independent Studies and Capstones, Program in Audiology and Communication Sciences, Washington University School of Medicine. Recuperado el 20 de febrero de 2010 de http://digitalcommons.wustl.edu/pacs_capstones/256.

Rendón, M. (2007). Regulación emocional y competencia social en la infancia. Revista Diversitas. Perspectivas en Psicología, 3, 349-361.

Skliar, C., Massone, M. y Veinberg, S. (1995). El acceso de los niños sordos al bilingüismo y biculturalismo. Infancia y aprendizaje, 69(70), 85-100. Reimpreso en Ecos fonoaudiológicos, 2, 32-50

Tabachnick, B. y Fidell, L. (2001). Using multivariate statistics (4th ed.). New York: Harper \& Row.

Triado, I. (1991.) El desarrollo de la comunicación en el niño sordo. Revista Logopedia, Foniatría, Audiología, 11(3), 122-129.

Trianes, M. V., Blanca, M. J., Muñoz, A., Garcia, B., Cardelle-Elawar, M., e Infante, L. (2002). Relaciones entre evaluadores de la competencia social en preadolescentes: profesores, iguales y autoinforme. Anales de psicología, 18(2), 197-214.

Trianes, M. V., Cardelle-Elawar, M., Blanca, M. J. y Muñoz, A. (2003). Contexto social, género y competencia social autoevaluada en alumnos andaluces de 11-12 años. Electronic Journal of Research in Educational Psychology, 1(2), 37-56. 
Urbano, A. y Estruch, R. (1996). Trastornos de la audición y del equilibrio. En FarrerasRozman, Manual de Medicina Interna (13a ed.) (pp. 1393-1397) [CD-ROM]. Barcelona: Mosby Doyma Libros.

Wauters, L. y Knoors, H. (2007). Social integration of deaf children in inclusive settings. Journal of Deaf Studies and Deaf Education, 28(2), 1- 16. 